\title{
STUDENT FEEDBACK AND PROBLEM DEVELOPMENT FOR WEBWORK IN A SECOND-YEAR MECHANICAL ENGINEERING PROGRAM
}

\author{
Agnes G. d'Entremont ${ }^{1}$, Patrick J. Walls ${ }^{2}$, and Peter A. Cripton ${ }^{1}$ \\ Departments of ${ }^{1}$ Mechanical Engineering and ${ }^{2}$ Mathematics, University of British Columbia \\ agnes.dentremont@mech.ubc.ca, pwalls@math.ubc.ca, cripton@mech.ubc.ca
}

\begin{abstract}
WeBWorK is a widely-used open-source, online homework tool where instructors may author their own problems, or select problems from an Open Problem Library. While it is extensively and globally used in mathematics, there are few problems available for engineering subjects. Due to initial student feedback based on mathematics problems, we decided to compare WeBWorK directly to our Blackboard Learn LMS for online homework during an integrated second-year Mechanical Engineering program.

Students were assigned two problem sets in Blackboard and two problem sets in WeBWorK, and then completed a survey. Results show a strong preference for WeBWorK in all areas, including ease of use, ease of navigation, clear feedback, reported enhancement of learning, etc.

We outline the primary benefits and drawbacks of using WeBWorK, and conclude by recommending WeBWorK for online homework in engineering courses.
\end{abstract}

Keywords: WeBWorK, online homework, learning management system, open-source, mechanical engineering

\section{INTRODUCTION}

As part of our second-year Mechanical Engineering program at the University of British Columbia, several hundred homework questions have been implemented in our learning management system (LMS), Blackboard Learn (Test tool), for eight academic subjects. During the 2015-16 school year, in addition to LMS-based questions, students were exposed to both a paid textbook publisher homework site and WeBWorK, an open-source, online homework system. Unsolicited, the students expressed a strong preference for WeBWorK over the other options in feedback sessions. As a result, we started exploring WeBWorK as a possible alternative platform to our LMSbased online homework problems.

We chose to compare WeBWorK to Blackboard Learn directly by assigning two problem sets on each platform and surveying students (2016-17 cohort). In this paper, we discuss how WeBWorK functions for instructors and students (particularly compared to Blackboard), the outcome of the comparison between the two platforms, and the advantages and disadvantages of each system.

\section{BACKGROUND}

WeBWorK is an open-source, online homework tool used by more than 1000 institutions globally [1], primarily for mathematics courses, and it has an associated Open Problem Library (OPL) of about 35,000 problems that are free for instructors to use, modify, and add to [2].

\subsection{Research about online homework tools}

Online homework tools provide a number of benefits. The primary benefit to students is automated (often immediate) feedback on their work, allowing them to correct errors in understanding and methodology. For instructors, we can provide many types of questions (e.g. multiple choice, calculated answer), assign different variable sets to each student (to limit copying), automate scoring and grade recording, and access summary statistics on class success for each problem or topic.

While several studies indicate performance improvements or equivalence when using online homework tools versus pen and paper [3]-[5], there are few comparisons of the various online tools that are available. One study asked students to compare WeBWorK to "other homework systems" they had used, and showed a student preference for WeBWorK [3], but did not directly compare systems which all the students had used.

Current engineering content available in the WeBWork OPL is limited. As of writing, the engineering subject areas listed are Electric Circuits (99 problems), and Statics and Mechanics of Materials (72 problems), with a Thermodynamics set (87 problems) pending release [6]. 
There are two basic ways for instructors to use WeBWorK: selecting existing problems from the OPL, or coding new problems in a Perl-based language, which can reside locally or be uploaded to the OPL. Since the current OPL problems are primarily in math, we coded two local problem sets covering four engineering science topics, and added math questions to each set from the OPL.

\subsection{Options for online homework}

Our focus is online homework tools that typically involve calculated answer questions, where students are randomly assigned different variable sets for the same problem, and where the system automatically grades each question by comparing the entered answer to a formula. This can provide instant feedback to students on their understanding of the material. Several options exist for instructors who wish to use online homework tools of this type.

Paid sites, such as those associated with textbook publishers, can be used to assign online homework in engineering courses (e.g. McGraw-Hill Connect [7], WebAssign [8], MasteringEngineering [9]). These sites have existing problems available, so the instructor does not have to create or code problems, and the problems may be coordinated with the course text in aspects such as notation, problem numbering, and so on. They may also be very well tested, so there are few question errors and may have eliminated combinations of variable values that cause special cases which don't work. However, there is often a substantial cost for students, and instructors do not typically have the ability to modify questions.

Instructors can also implement problems within their LMS (e.g. Blackboard Learn, Desire2Learn). This involves creating each problem the instructor wishes to assign, unless formal or informal sharing of question pools is implemented at an institution or the instructor has an importable test bank from a publisher. There is no straightforward means to share these questions with the larger educational community. There is typically limited testing completed before deployment to students and there may be variable combinations of question parameters that cause nonsensical results. These combinations are often difficult to find in advance of release.

Finally, WeBWorK is an open, online homework system with integrated problem bank (OPL) which is free for students. Instructors can select existing questions without modifications, modify existing questions, or code their own new questions (which can be uploaded to the OPL to share with others or can reside locally). The testing of existing questions may vary depending on whether the question was used extensively with classes prior to release, so some of the issues seen in an LMS implementation can be present here as well, even without authoring new questions.

\subsection{Problem authoring and coding in WeBWorK}

A WeBWorK problem is simply a text file written in $\mathrm{PG}$, a perl-based programming language initially developed in 1995 at the University of Rochester and now supported by the Mathematical Association of America (MAA). There are many resources provided by the MAA introducing $\mathrm{PG}$ and its extensions however the most common technique for authoring new problems is to modify an MAA template or an existing PG file from the OPL [10].

Development and testing of WeBWorK problems usually takes place within an instance of the WeBWorK web application. This means that PG code is written, modified, rendered and tested all within the WeBWorK application. The development of WeBWorK problems is more flexible than problems created within an LMS application because it is written in its own language with a rich set of datatypes to express any kind of mathematical expression (real, complex, formula, vector, matrix, list, set, interval). In addition, since WeBWorK problems are rendered in the browser, there is simple PG code to include images, links and mathematical notation (LaTeX).

Problems can be set up with variables that display as specific numbers in the problem text and have defined ranges. WeBWorK allows multiple equations to be used to obtain the final answer, which makes creating a final answer formula straightforward. The code can use inequalities and Boolean statements to check for and remove problem combinations of specific variable values, and a range of acceptability (e.g. $+/-5 \%,+/-0.01$, etc.) can be set for an answer.

The flexibility of the PG code means that problems can be set-up in a number of ways, including with a series of staged answer boxes. For example, for a projectile motion question, a WeBWorK problem could have answer boxes for both time to reach maximum height and resulting horizontal distance. Each student answer is shown as correct (green) or not correct (red) independently. This can allow students to determine at what stage of the problem they have gone off track.

The WeBWorK application and the OPL are open source projects, and are hosted on GitHub. Anyone can develop problems and contribute to the OPL simply by creating a pull request to the OPL GitHub repository (see https://github.com/openwebwork).

\subsection{Using WeBWorK}

When a student logs in to their WeBWorK course, they see a list of links to assigned problem sets as well as the assignment due dates. Clicking on a problem set brings the student to a list of links to individual problems in the problem set. Students can begin working on questions sequentially or navigate to any particular question. 
Every student attempt per problem is saved and students can revisit a question any time before the assignment due date. Students may submit a single attempt, multiple attempts or infinite attempts depending on the setting set by the instructor. Students may check whether the syntax they have used produced the answer they intended before submitting an attempt. When students submit their attempt for a question, the WeBWorK answer parser determines if the attempt is correct and displays a table of information to the student showing the raw student answer, the student answer rendered in LaTeX and whether it is correct (green) or incorrect (red).

The immediate feedback and the easy navigation through the problem sets are features that distinguish the WeBWorK student user interface.

\subsection{Problem authoring in Blackboard Learn}

Authoring calculated answer problems in Blackboard Learn involves creating tests, as there is no stand-alone homework tool that allows these types of questions. The interface is a series of input fields on the pages of the Create/Edit Calculated Formula Question tool, which provides a very low barrier to begin authoring, unlike learning syntax for WeBWorK.

The question is written with variables defined by square brackets and a variable name (e.g. [mass], [q], etc.). These variables can then be used in a single equation to define the answer. This can result in very complex equations that are difficult to debug when several formulae have been used to arrive at the final result. A range of values may then be input for each variable and the total number of variable sets that will be created can be defined. A range of acceptability (e.g. +/$5 \%,+/-0.01$, etc.) can be set for an answer. Images may be uploaded and added to the questions.

\subsection{Using Blackboard Learn}

Students being a problem set by clicking on the test link to that problem set. They are shown an initial page of details about the test/problem set (this is set up to include information about test time limits and other setting not used in the homework context), and can click to advance to the questions. There are several ways to configure the test, but the most logical is to present all questions on one page, so students can see all the problems they must solve without requiring a linear navigation through them.

Questions show the question text with specific variable values and a single answer box, as well as the point value of each question. Students may save answers to individual questions, but can only submit once per attempt, so they must necessarily work on all questions prior to any feedback. Answer syntax is cannot be checked prior to submission. Once submitted, a confirmation page is shown. Students must then navigate elsewhere to see what questions were correct or incorrect, if that information is made available prior to the due date. If multiple attempts are permitted, the value for variables in the questions change with each new attempt.

\section{METHODS}

We compared WeBWorK in an integrated, teamtaught engineering science and math course of 124 second-year Mechanical Engineering students (MECH 221). The course covers five subject areas (dynamics, electric circuits, solid mechanics, materials, and differential equations). Students are assigned 10 problem sets (over 10 weeks), and each problem set includes problems from every subject area. The problem sets had been previously implemented using our LMS, Blackboard Learn, however co-instructors from Mathematics over the past year have elected to remove the math problems from the Blackboard problem sets and assign parallel WeBWorK problems for each set. As a result, only engineering science problems were compared between the two systems.

\subsection{Homework releases}

The first two homework sets of the course (problem sets PS1 and PS2) were released in their existing form on the course LMS site using the Blackboard Learn test tool, which was the method available for implementing calculated answer questions.

We implemented the next two homework sets (problem sets PS3 and PS4) using WeBWorK. The default setting for WeBWorK problems is to allow unlimited attempts for each problem, which was unlike the Blackboard homework sets (which allowed a single attempt). We limited students to two attempts for multiple choice questions, while allowing unlimited attempts at calculated answer questions. WeBWork is also designed to give immediate feedback (correct/incorrect) on the problem page and allow students to attempt the problem again without navigating elsewhere. The Blackboard sets were configured to show feedback, but only after the due date. Feedback was shown on a separate page.

PS3 included 11 newly-coded engineering science questions, specifically 4 multiple choice and 7 calculated answer (plus 9 math questions from the OPL). PS4 included 8 newly-coded engineering science questions, specifically three multiple choice and five calculated answer (plus 5 math questions). To replicate the questions blocks in Blackboard that allowed randomly assigning questions, we created several problem sets with different multiple choice questions which were assigned to blocks of students.

We subsequently released two more Blackboard-based homework sets (PS7 and PS8) with settings as close to WeBWorK as possible, through the following changes: 
- Allowing unlimited attempts for each homework set (with the average mark assigned). Note that it was not possible to set number of attempts by question, which means that multiple choice questions can always be answered correctly within a few attempts

- Allowing students to see which problems they got right or wrong immediately (as opposed to after the due date), and to see the correct answers for each attempt after the due date

Each Blackboard attempt required students to complete all questions again, even those they answered correctly the first time. Each attempt also changed the specific numbers a student was given for the calculated answer questions with variables, meaning that they had to recalculate numerical answers even for questions that they originally got correct. Finally, because some problem sets involved randomly assigning problems from a set of questions, student may be given new problems they had not yet seen on their reattempt.

\subsection{Survey and feedback}

We surveyed students about their preferences between online homework systems based on the first two
Blackboard homework sets and the two WeBWorK homework sets. We also asked them about the importance of certain aspects in selecting a new online homework system.

Following the changes to the Blackboard homework (to attempt to mimic WeBWorK), students were invited to provide informal feedback.

\section{RESULTS}

There were 82 responses to the survey (out of 124 students), and questions included between 74 and 82 responses (some respondents did not answer all questions).

Students rated WeBWorK higher than the LMS system for every aspect included in the survey, including ease of use, clarity of feedback, and more (Table 1). They also agreed with statements that WeBWorK enhanced their learning, and they would like to use it in the future. The only two statements where the Blackboard tool received mean ratings above neutral (in the agreement range) were about navigation of the homework sets.

Table 1: Survey results about online homework tools following two Blackboard homework sets and two WeBWork homework sets. Based on a five-point scale where 1 was strongly disagree, 3 was neutral, and 5 was strongly agree.

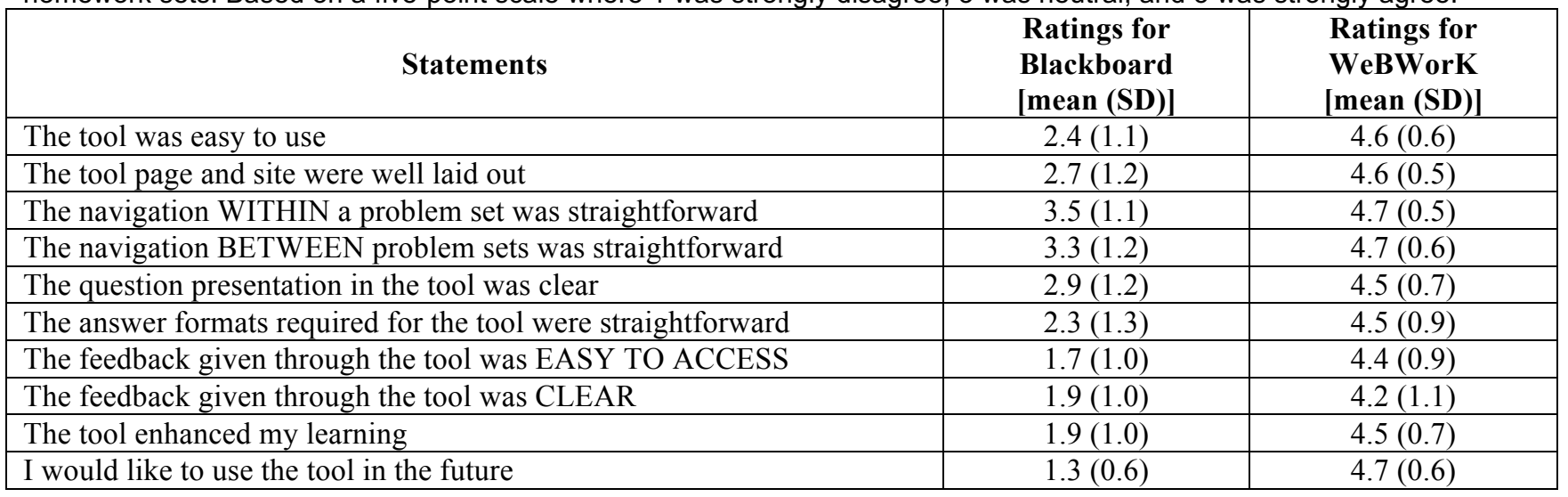

When asked directly to select one option, students overwhelmingly preferred WeBWorK $(92 \%)$ compared to Blackboard $(0 \%)$, with $4 \%$ each responding they were not sure or preferring a different system.

When asked about the qualities for a new online homework tool, students found ease of use and having a common tool across courses to be the most important aspects (Table 2).

Here are some representative comments from the students on WeBWorK as a homework tool:

- "Relative to [Blackboard Learn], it is a way better tool. Even if the opportunities to answer are limited to a few tries, it is still much more helpful in terms of figuring out what parts of your solution you need to fix in order to learn how to do the problem properly."

- "I think webwork is effective as a homework tool because it provides feedback instantaneously which allows me to learn from my mistakes as [I] work through the problems."

- "Because I could try multiple times I would try different approaches and developed new techniques that helped me on the tests."

- "The ability to preview an answer is valuable to fix syntax errors."

- "Much better system, most new physics problems take me a couple of tries of trial and error, and if I 
am not given marks until I use the correct approach, I will continue trying much more."

- "Webwork helps me learn as I do the problems."

Table 2: Survey results about importance of various aspects or qualities in selecting a new online homework system. Based on a 5-point scale where 1 was strongly disagree, 3 was neutral, and 5 was strongly agree.

\begin{tabular}{|l|c|}
\hline \multicolumn{1}{|c|}{ Statements } & $\begin{array}{c}\text { Ratings } \\
\text { [mean (SD)] }\end{array}$ \\
\hline The tool is open source & $3.8(0.9)$ \\
\hline The tool is easy to use & $4.6(0.6)$ \\
\hline $\begin{array}{l}\text { The tool builds a greater engineering } \\
\text { academic community }\end{array}$ & $3.9(1.0)$ \\
\hline $\begin{array}{l}\text { The tool is common among all my } \\
\text { courses/across UBC }\end{array}$ & $4.2(0.9)$ \\
\hline $\begin{array}{l}\text { The tool is part of a larger learning } \\
\text { management tool }\end{array}$ & $3.8(1.0)$ \\
\hline
\end{tabular}

To explore the usefulness of feedback and additional attempts, we examined attempt statistics from PS7 and PS8 (Blackboard, unlimited attempts) compared to PS3 and PS4 (WeBWorK). Due to differences in the systems, the attempts were reported in different ways. For Blackboard problems sets PS7 and PS8, there was a median of 1 attempt per student for the whole problem set, and a mean of 2.1 (PS7) and 1.7 (PS8) attempts per student. Another notable result was that students did not always increase their mark in subsequent attempts, likely due to changing questions and changing number values within the questions. The median number of attempts per calculated answer engineering science question for WeBWorK problem sets PS3 and PS4 were between 2 and 16 attempts per student, depending on the particular question, with high percentages $(67-100 \%$, depending on the question) of students ultimately getting the correct answer. Since it is possible that there was less student engagement with problem sets as the term progressed, we examined the statistics for later sets where we had coded at least some of the engineering science problems. In PS9 and PS10 (the last two homework sets, coded in WeBWorK), the median attempts per calculated answer engineering science question were between 2 and 8 attempts per student.

After adjusting the Blackboard problem sets to provide unlimited attempts, several students commented that it was better, but did not match WeBWorK in ease of use. For example, students expressed some frustration with having to repeat questions they had already answered correctly to reattempt some questions.

\section{DISCUSSION}

Students clearly preferred WeBWorK over Blackboard Learn for the online homework in our course.
Specific aspects cited were clear feedback (including syntax feedback), better user interface, repeated attempts (same random numbers), and other features not available with Blackboard.

Besides the direct student responses, the number of attempts students made when offered unlimited tries in each platform differed greatly, with students making more attempts with WeBWorK. While we must interpret this cautiously, both because the measure was different between systems (by problem or by problem set), and because there were some coding errors in the initial WeBWorK problems (corrected after release) that may have spurred students to high numbers of attempts, still this may be an indication that WeBWorK encourages students to spend time figuring out the correct answer if they have gotten a question wrong. As an aside, one student said specifically that they would prefer WeBWorK problem sets with errors over error-free Blackboard-based problem sets.

Substantial inconveniences remained with the adjusted Blackboard-based problem sets, which required completing the entire problem set again (sometimes with new questions or values) to reattempt one problem. Informal feedback suggested that this alteration was not sufficient to match the experience of using WeBWorK.

There were several limitations to this comparison. Because of the differing options available on the two platforms, there were some substantial differences in the delivery of the problem sets. Other limitations include not formally asking for feedback on the Blackboard problem set changes, and having several coding errors in the WeBWorK problem sets (about 1-2 errors per set, however some were caught on the first day of release) that may have inflated the number of the attempts for specific problems. Math problems were assigned solely in WeBWorK during the course, and drawn from the OPL it is possible that the additional exposure, including during PS1 and PS2 could have influenced the results.

\subsection{Advantages and drawback for instructors}

We identified a range of positives and negatives for instructors using WeBWorK in engineering classes.

Advantages include:

- Access to free, open-source problems in a growing variety of subjects in the OPL

- Flexibility in the presentation of problems and the structure of student answers when coding

- Being a purpose-built homework tool, with substantial control in assigning problem sets and collecting student data

- Can interface with LMS to pull in class lists

- Can import local problems shared between instructors but not posted to the OPL 
Drawbacks include:

- A significant investment of time is required to learn the coding and create problems

- There are few existing WeBWorK problems in engineering subjects

- Possibility of errors or flawed variable combinations in OPL problems; may be less likely with a paid system

- In our case, no direct or automatic import of homework grade data into the LMS grade book

- No simple method to randomize questions to students, if drawing a sample from a question set

\subsection{Advantages and drawback for students}

Similarly, we considered the positives and negatives of using WeBWorK for the students.

Advantages include:

- No cost to students

- Clear feedback (red or green), and students can immediately attempt the problem again without navigating elsewhere

- Easy navigation within and between problem sets

- Can check syntax before submitting answer

- Permits multiple answer boxes within a question (e.g. for multiple intermediate steps in a response), and will show correct or incorrect for each box, so students can see where they started to go wrong

- Used frequently within Math departments, so students may be familiar with the tool already

Drawbacks include:

- Uses a second online system, in addition to LMS

Overall, despite the identified drawbacks, the flexibility of the system, OPL problem sharing, and preferred student interface lead us to recommend using WeBWorK in engineering courses.

Since we will be converting the remainder of the problem sets, we will have some additional data about identical problems that were completed in Blackboard this year and will be completed in WeBWorK next year.

Our problems will be released to the OPL once further testing has been completed and a systematic taxonomy for problem categorization has been finalized and applied.

\section{CONCLUSIONS}

Students in our second-year integrated Mechanical Engineering program rated WeBWorK higher than the Blackboard Learn-based online homework on every metric, and overwhelmingly preferred WeBWorK in a direct comparison.
Current WeBWorK OPL problems are primarily in math, with limited engineering problems available. We see an opportunity to develop problems on this platform for use in our program and to share with the greater engineering community. If others contribute, we could eventually build a large selection of crowd-sourced OPL engineering problems.

\section{Acknowledgements}

We would like to acknowledge the critical support provided by Jim Sibley (UBC Faculty of Applied Science Centre for Instructional Support), and Jenny Song for her coding work. We would also like to acknowledge the colleagues and teaching assistants who tested problems, and the students who participated in the survey.

\section{References}

[3] M. K. Swanbom, D. W. Moller, K. Evans, and T. Reeves, "Open-source, online homework for statics and mechanics of materials using WeBWorK: assessing effects on student learning," ASEE Annu. Conf., 2016.

[4] L. Hirsch and C. Weibel, "Statistical Based Evidence that Web-Based Homework Helps," MAA Focus, vol. 23, no. 2, p. 14, 2003.

[5] K. Evans, P. Hummel, and M. Gates, "An expanded study to assess the effect of online homework on student learning in a first circuits course," ASEE Annu. Conf., 2016.

[6] K. Evans, "Personal communication." 2017.

[7] "McGraw-Hill Connect." [Online]. Available: http://connect.mheducation.com/. [Accessed: 25Apr-2017].

[8] “WebAssign.” [Online]. Available: https://webassign.com/. [Accessed: 25-Apr-2017]. "MasteringEngineering." [Online]. Available: http://www.pearsonmylabandmastering.com/north america/masteringengineering/. [Accessed: 25Apr-2017].

[10] "Problem Authoring Background Information WeBWorK." [Online]. Available: http://webwork.maa.org/wiki/Problem_Authoring Background_Information. [Accessed: 05-May2017]. 